\title{
Enhanced Plasmid DNA Transfer into Tumor Cells by Nanoparticle Composed of Cholesteryl Triamine and Diamine
}

\author{
Yoshiyuki Hattori, ${ }^{* a}$ Tsukasa Nakamura, ${ }^{a}$ Hiroaki Ohno, ${ }^{b}$ Nobutaka Fujii, ${ }^{b}$ and Yoshie Maitani ${ }^{a}$ \\ ${ }^{a}$ Institute of Medicinal Chemistry, Hoshi University; 2-4-41 Ebara, Shinagawa-ku, Tokyo 142-8501, Japan: and \\ ${ }^{b}$ Graduate School of Pharmaceutical Sciences, Kyoto University; Sakyo-ku, Kyoto 606-8501, Japan. \\ Received December 12, 2012; accepted February 4, 2013
}

Previously, we prepared cationic nanoparticles (NP and NP-N) composed of cholesteryl diamine (OHChol, (3S)- $\mathrm{N}$-(2-(2-hydroxyethylamino)ethyl)cholesteryl-3-carboxamide) and cholesteryl triamine (OH-NChol, (3S)- $\mathrm{N}$-(2-(2-(2-hydroxyethylamino)ethylamino)ethyl)cholesteryl-3-carboxamide), respectively, with Tween 80 for small interfering RNA (siRNA) delivery into tumor cells. In this study, we prepared NP-0.25N composed of $\mathrm{OH}-\mathrm{Chol}$ and $\mathrm{OH}-\mathrm{N}-\mathrm{Chol}$ at a molar ratio of $3 / 1$ with Tween 80, and evaluated the transfection efficiency of plasmid DNA (pDNA) into tumor cells. NP-N exhibited lower transfection activity than NP; however, NP-0.25N showed higher transfection activity than both NP and NP-N in various tumor cells. NP$0.25 \mathrm{~N}$ increased the amount of internalized pDNA by increased cellular association, and improved the escape from endosomes after clathrin-mediated endocytosis. The results of the experiments suggested that cholesteryl triamine may have potential as a helper lipid to increase the transfection for pDNA delivery by cationic cholesterol-based nanoparticles.

Key words cationic nanoparticle; plasmid DNA delivery; transfection; cholesteryl diamine; cholesteryl triamine; tumor cell

Cationic liposome or nanoparticle-mediated transfer of plasmid DNA (pDNA) is a promising approach for gene delivery. Cationic lipids and improved formulations of liposome and nanoparticle have been developed for the efficient delivery of pDNA into cells. For efficient gene delivery to tumor tissue, the pharmacokinetics and intracellular trafficking of pDNA after endocytosis must be controlled. To overcome poor endosomal escape and subsequent degradation of pDNA in late lysosomes, $\mathrm{pH}$-sensitive fusogenic peptides and lipids have been developed as components of liposomal pDNA vectors. ${ }^{1,2)}$

Previously, we reported that cationic nanoparticles composed of cholesteryl diamine ((3S)- $N$-(2-(2-hydroxyethylamino)ethyl)cholesteryl-3-carboxamide, $\mathrm{OH}-\mathrm{Chol})$, having a hydroxyethyl group at the amine headgroup and an amido-linker (Fig. 1), achieved high transfection ability for pDNA delivery. ${ }^{3)}$ Polyethylenimines (PEIs) are used as a pDNA vector and have been shown to have the ability to escape the endosome by buffering capacity. ${ }^{4}$ Therefore, we recently synthesized cholesteryl triamine ((3S)- $N$-(2-(2-(2-hydroxyethylamino)ethylamino)ethyl)cholesteryl-3-carboxamide, OH-N-Chol) (Fig. 1), which has two units of aminoethylene between the amino head group and the cholesterol skeleton, to facilitate the endosomal escape of nucleic acid transfected by OH-N-Chol-based nanoparticles. ${ }^{5)}$ In this study, we optimized the formulation of cationic cholesterol derivative-based nanoparticles for pDNA delivery. Here, we found that the inclusion of $\mathrm{OH}-\mathrm{N}-\mathrm{Chol}$ into $\mathrm{OH}-\mathrm{Chol}-$ based nanoparticles could increase transfection activity in tumor cells.

\section{MATERIALS AND METHODS}

Plasmid DNA The plasmid pCMV-luc encoding the firefly luciferase gene under the control of the cytomegalovirus (CMV) promoter was constructed as previously described.6) Fluorescein isothiocyanate (FITC) labeling for pDNA was

The authors declare no conflict of interest. performed using the protocol of the Label $\mathrm{IT}^{\circledR}$ Nucleic Acid Labeling Kit, Fluorescein (Mirus, Madison, WI, U.S.A.).

Preparation and Size of Nanoparticles and Nanoplexes Cholesteryl diamine, (3S)- $N$-(2-(2-hydroxyethylamino)ethyl)cholesteryl-3-carboxamide (OH-Chol), and cholesteryl triamine, (3S)- $N$-(2-(2-(2-hydroxyethylamino)ethylamino)ethyl)cholesteryl-3-carboxamide (OH-N-Chol), were synthesized as previously reported. ${ }^{5,7)} \mathrm{NP}$ and NP-N consisted of $1 \mathrm{mg} /$ $\mathrm{mL} \mathrm{OH}-\mathrm{Chol}$ and $\mathrm{OH}-\mathrm{N}-\mathrm{Chol}$ as a cationic lipid, respectively, with $5 \mathrm{~mol} \%$ Tween 80 (Table 1). Formulation of NP- $0.25 \mathrm{~N}$ involved substitution of $25 \% \mathrm{OH}-\mathrm{Chol}$ with that of NP with equimolar OH-N-Chol (Table 1). Each nanoparticle was prepared by a dry-film method. The nanoparticle/pDNA complex<smiles>CC(C)CCC[C@H](C)[C@H]1CCC2C3CC=C4CC(C(=O)NCCNCCO)CCC4(C)C3CCC21C</smiles>

\section{OH-N-Chol}

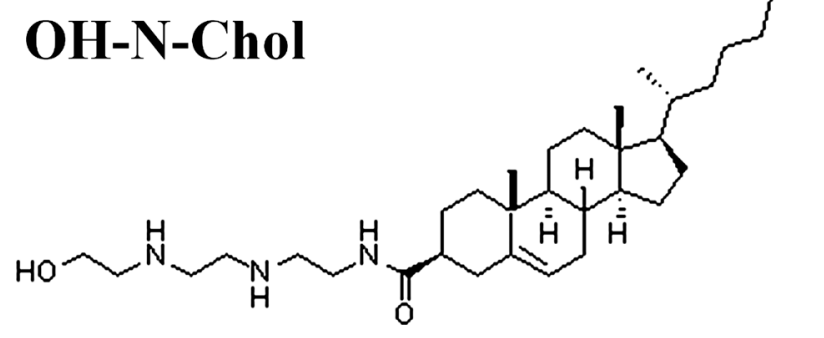

Fig. 1. Structure of Cationic Cholesterol Derivatives: Cholesteryl Diamine, (3S)- $N$-(2-(2-Hydroxyethylamino)ethyl)cholesteryl-3-carboxamide (OH-Chol); Cholesteryl Triamine, (3S)-N-(2-(2-(2-Hydroxyethylamino) ethylamino)ethyl)cholesteryl-3-carboxamide (OH-N-Chol) 
Table 1. Particle Size and $\zeta$-Potential of Nanoparticles and Nanoplexes

\begin{tabular}{|c|c|c|c|c|c|c|}
\hline & \multirow{2}{*}{ Formulation } & \multirow{2}{*}{$\mathrm{mol}^{\%} \mathrm{O}$} & \multicolumn{2}{|c|}{ Nanoparticle $\mathrm{e}^{a)}$} & \multicolumn{2}{|c|}{ Nanoplex ${ }^{a, b)}$} \\
\hline & & & Size $(\mathrm{nm})$ & $\zeta$-Potential $(\mathrm{mV})$ & Size (nm) & $\zeta$-Potential $(\mathrm{mV})$ \\
\hline NP & OH-Chol/Tween 80 & $95 / 5$ & $900 \pm 19.5$ & $50.8 \pm 1.4$ & $948 \pm 21.8$ & $48.8 \pm 4.2$ \\
\hline $\mathrm{NP}-0.25 \mathrm{~N}$ & OH-Chol/OH-N-Chol/Tween 80 & $71 / 24 / 5$ & $526 \pm 8.2$ & $49.8 \pm 0.6$ & $868 \pm 98.8$ & $45.7 \pm 0.2$ \\
\hline NP-N & OH-N-Chol/Tween 80 & $95 / 5$ & $1170 \pm 3.8$ & $50.6 \pm 1.1$ & $834 \pm 53.0$ & $36.5 \pm 0.8$ \\
\hline
\end{tabular}

$a$ ) In water. $b$ ) Charge ratio $(+/-)$ of nanoparticle/DNA $=3: 1$. Values represent means \pm S.D. $(n=3)$.

(nanoplex) at various charge ratios $(+/-)$ of cationic lipid to pDNA was formed by the addition of each nanoparticle to $2 \mu \mathrm{g}$ of pDNA with gentle shaking and leaving at room temperature for $10 \mathrm{~min}$. The charge ratio $(+/-)$ of nanoparticles/ pDNA is expressed as the cationic lipid/DNA phosphate ratio (mol). The particle size distributions and $\zeta$-potentials were measured using ELS-Z2 (Otsuka Electronics Co., Ltd., Osaka, Japan) at $25^{\circ} \mathrm{C}$ after diluting the dispersion to an appropriate volume with water.

Luciferase Assay Human prostate tumor PC-3, human cervical carcinoma HeLa, human lung mucoepidermoid carcinoma H292, and murine colon carcinoma Colon 26 cells were plated into 6-well culture dishes at a density of $3 \times 10^{5}$ cells per well. For transfection, each nanoplex of $2 \mu \mathrm{g}$ of pCMV-luc formed at the indicated charge ratio $(+/-)$ was diluted in $1 \mathrm{~mL}$ of medium supplemented with $10 \%$ fetal bovine serum (FBS) and then incubated with the cells for $24 \mathrm{~h}$. Lipofectamine 2000 lipoplexes (Invitrogen Corp., Carlsbad, CA, U.S.A.) were prepared for transfection according to the manufacturer's protocol. Luciferase expression was measured as counts per sec (cps) $/ \mu$ g protein using the luciferase assay system (PicaGene, Toyo Ink Mfg. Co., Ltd., Tokyo, Japan) and bicinchoninic acid (BCA) reagent (Pierce, Rockford, IL, U.S.A.) as previously reported. ${ }^{3)}$

Gel Retardation Assay One microgram of pDNA was mixed with aliquots of nanoparticles ( 1 to 4 charge equivalents of nanoparticles). After $10 \mathrm{~min}$ incubation of the nanoplexes in water, they were analyzed by $1.5 \%$ agarose gel electrophoresis in Tris-borate-ethylenediaminetetraacetic acid (EDTA) (TBE) buffer and visualized by ethidium bromide staining as previously reported. ${ }^{7)}$

Flow Cytometric Analysis PC-3 cell cultures were prepared by plating the cells on 6 -well culture dishes $24 \mathrm{~h}$ prior to each experiment. Each nanoparticle was mixed with $2 \mu \mathrm{g}$ of FITC-labeled pDNA at a charge ratio $(+/-)$ of $3 / 1$. For measurement of cellular association of the nanoplexes, the cells were transfected with NP, NP- $0.25 \mathrm{~N}$ or NP-N nanoplex, and then incubated for $3 \mathrm{~h}$. For investigation of the mechanism of cellular uptake with endocytosis inhibitors, the cells were treated with medium containing $400 \mathrm{~mm}$ sucrose, $50 \mu \mathrm{M} 5-(N-$ ethyl- $N$-isopropyl)amiloride (EIPA; Sigma Chemical Co., St. Louis, MO, U.S.A.) or $5 \mu \mathrm{g} / \mathrm{mL}$ filipin (Sigma Chemical Co.) for $30 \mathrm{~min}$. After the treatments, the cells were transfected with NP, NP- $0.25 \mathrm{~N}$ or NP-N nanoplex, in the presence of each inhibitor, and then incubated for $3 \mathrm{~h}$.

After the incubations, the amount of FITC-labeled pDNA in the cells was determined by examining fluorescence intensity on a FACSCalibur flow cytometer as previously described. ${ }^{8)}$

Confocal Microscopy PC-3 cell cultures were prepared by plating the cells at a density of $3 \times 10^{5}$ cells on $35 \mathrm{~mm}$ culture dishes $24 \mathrm{~h}$ prior to each experiment. Each nanoparticle

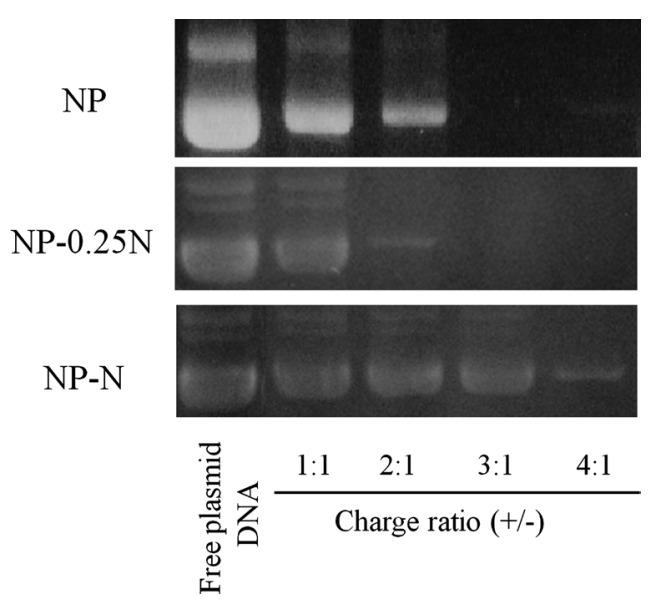

Fig. 2. Effect of Charge Ratio $(+/-)$ on DNA Association with Nanoparticles

Nanoplexes formed at various charge ratios $(+/-)$ were analyzed using agarose gel electrophoresis.

was mixed with $2 \mu \mathrm{g}$ of FITC-labeled pDNA at a charge ratio $(+/-)$ of $3 / 1$. The mixtures were diluted in $1 \mathrm{~mL}$ of medium supplemented with $10 \% \mathrm{FBS}$, and incubated with PC-3 cells. For observation of the intracellular localization of pDNA, after the 3-h incubation, the cells were fixed and stained with propidium iodide as previously reported.9) For evaluation of endosomal escape after internalization, after the 24-h incubation, acidic compartments like endosomes were labeled with Lysotracker ${ }^{\circledR}$ Red DND-99 (Invitrogen) as previously reported. ${ }^{5)}$ Examinations were performed with an LSM5 EXCITER confocal laser scanning microscope (Carl Zeiss, Thornwood, NY, U.S.A.).

Statistical Analysis The statistical significance of differences between mean values was determined by using Student's $t$-test.

\section{RESULTS AND DISCUSSION}

Many parameters including the nanoparticle/pDNA complex (nanoplex) size and charge ratio $(+/-)$ are known to affect transfection efficiency. ${ }^{10,11)}$ In pDNA transfection in vitro, large lipoplexes over $700 \mathrm{~nm}$ in mean diameter induced efficient transfection; in contrast, lipoplexes of less than $250 \mathrm{~nm}$ were inefficient. $^{12)}$ Therefore, in this study, we prepared largesized nanoplex (around $1 \mu \mathrm{m}$ ) for evaluation of transfection efficiency. NP and NP-N were composed of OH-Chol and $\mathrm{OH}-$ $\mathrm{N}-\mathrm{Chol}$, respectively, with Tween 80 (Table 1). The average sizes of NP and NP-N nanoplexes were about 950 and $830 \mathrm{~nm}$, respectively, when the nanoplexes were formed at a charge ratio $(+/-)$ of $3 / 1$ (Table 1$)$. The $\zeta$-potentials of NP and NP-N 
nanoplexes were about +49 and $+37 \mathrm{mV}$, respectively.

To optimize the charge ratio $(+/-)$ for pDNA transfection, we monitored the association of pDNA with each nanoparticle by gel retardation electrophoresis (Fig. 2). Beyond a charge ratio (+/-) of $3 / 1$ in NP, no migration was observed; however, in NP-N, slight migration of pDNA was still observed at a charge ratio $(+/-)$ of $4 / 1$. This result indicated that association of pDNA with NP-N was weaker than that with NP. Han et al. reported that polyethylene glycol (PEG)-polyaspartamide with an ethylenediamine unit as a side chain (PEG-PAsp(DET)) exhibited single protonation in the side chain at a physiological $\mathrm{pH}$ of 7.4 while exhibiting double protonations at an

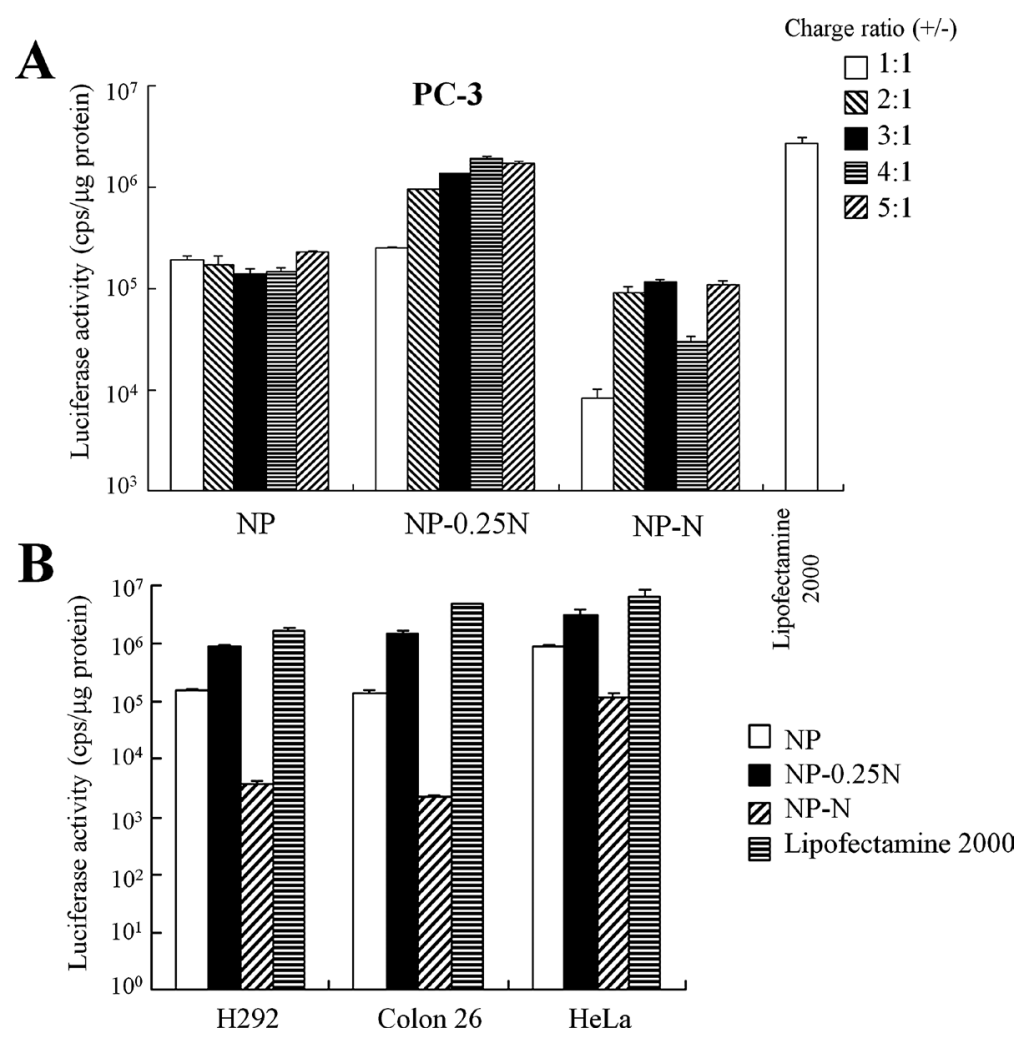

Fig. 3. Effect of Charge Ratio (+/-) on Transfection Activity in PC-3 Cells (A) and Comparison of Transfection Activities in H292, Colon 26 and HeLa Cells (B) at $24 \mathrm{~h}$ after Transfection by Nanoparticles

In A, NP, NP-0.25N and NP-N nanoplexes were prepared at various charge ratios (+/-). In B, NP, NP-0.25N and NP-N nanoplexes were prepared at a charge ratio $(+/-)$ of $3 / 1$. Each column represents the mean \pm S.D. $(n=4)$.
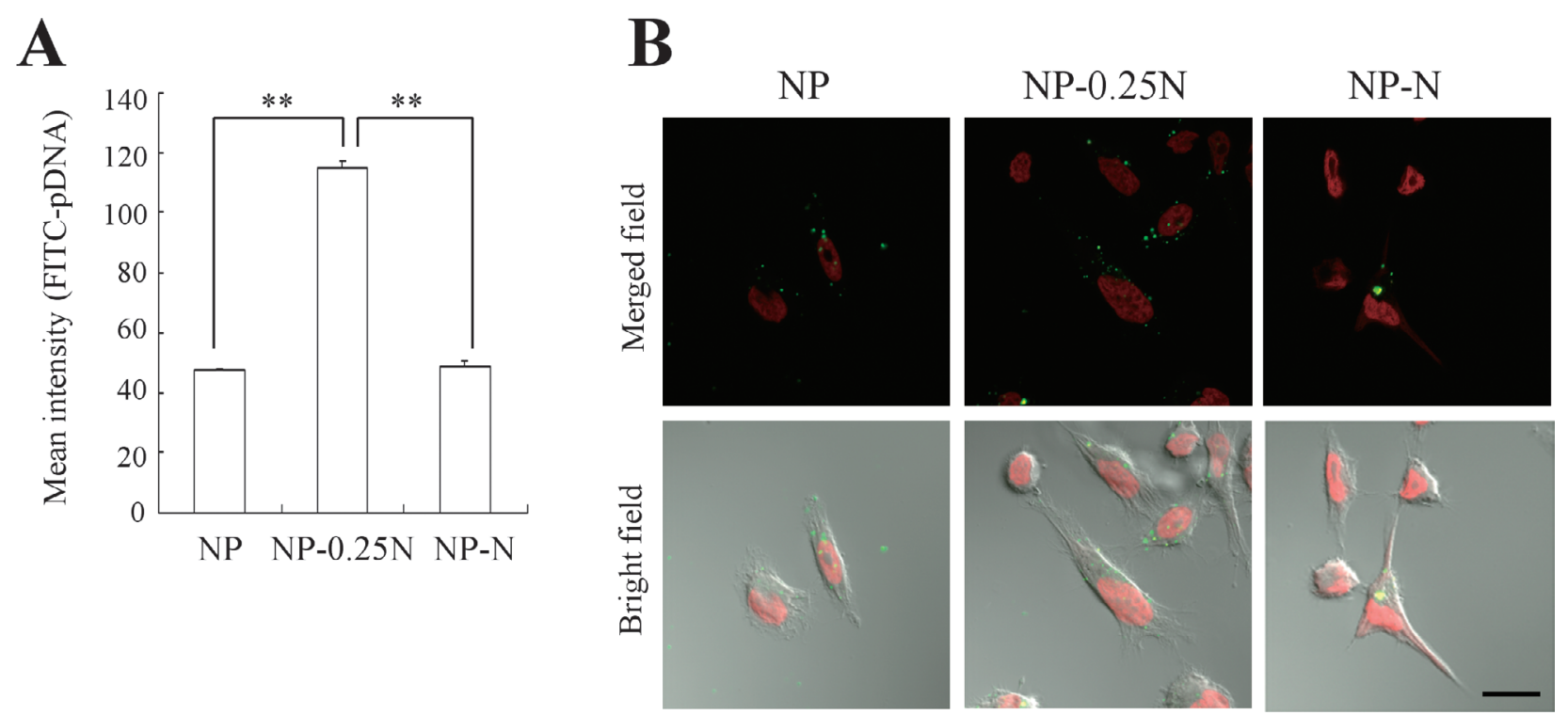

Fig. 4. Cellular Association (A) and Intracellular Localization (B) at $3 \mathrm{~h}$ after Transfection of Nanoplex

In $\mathrm{A}$, the association of nanoplex with PC-3 cells was determined on the basis of FITC-fluorescence by flow cytometry.** $p<0.01$. In $\mathrm{B}$, FITC-labeled pDNA in PC-3 cells was visualized by confocal microscopy. Green signals show localization of FITC-labeled pDNA, and red signals show localization of nuclei. Scale bar $=20 \mu$ m. 

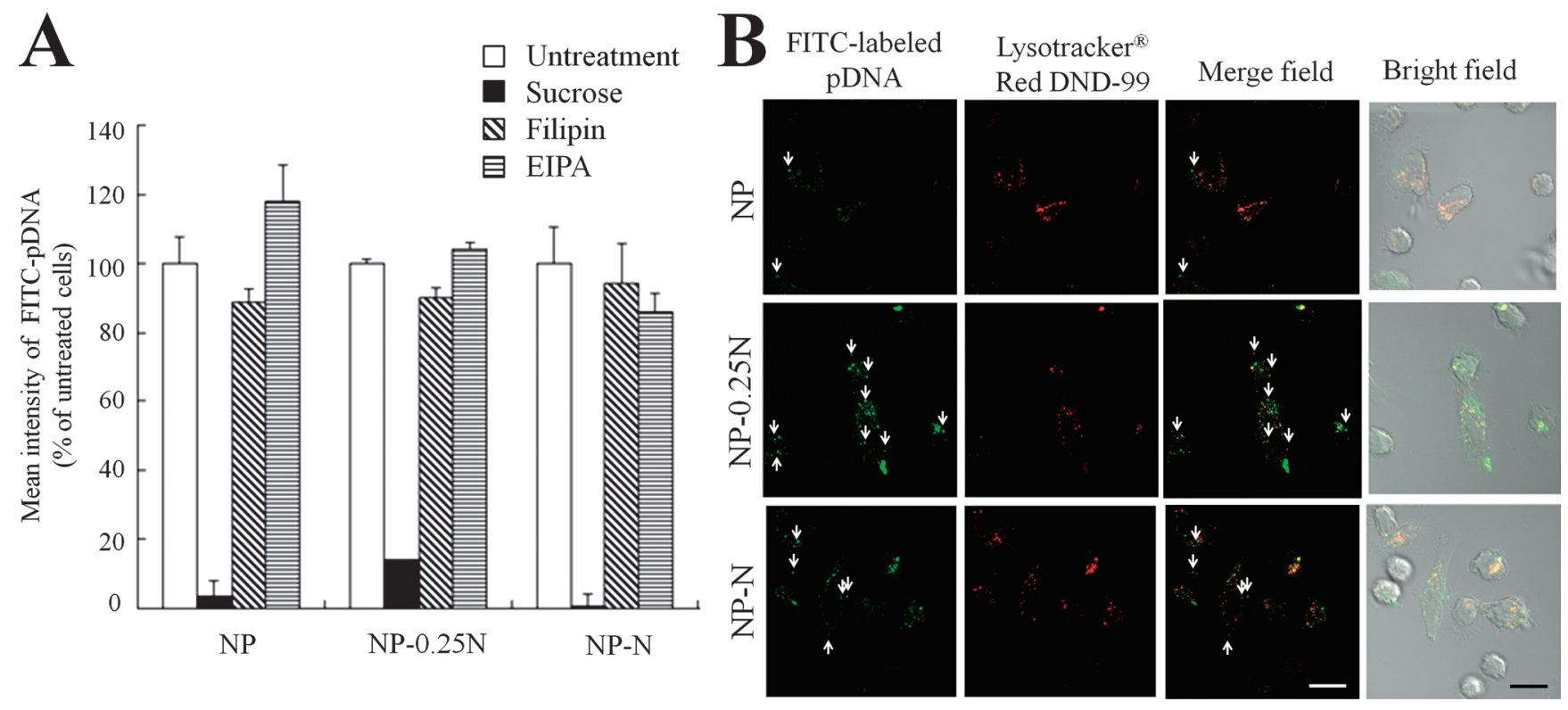

Fig. 5. Effect of Endocytosis Inhibitors on the Cellular Uptake of Nanoplex (A) and Endosomal Localization of FITC-Labeled pDNA through Nanoplex-Mediated Delivery (B) in PC-3 Cells

In A, nanoplexes of FITC-labeled pDNA were transfected into the cells in the presence of EIPA, filipin or sucrose for $3 \mathrm{~h}$. The cellular association of nanoplex was determined on the basis of FITC-fluorescence by flow cytometry. Each bar represents the mean \pm S.D. of three experiments. In B, nanoplexes of FITC-labeled pDNA were transfected into the cells. After 24-h incubation, acidic compartments like endosomes were stained with Lysotracker red DND-99. Localizations of FITC-labeled pDNA and endosomes in the cells were visualized by confocal microscopy. Green signals show the localization of FITC-labeled pDNA, and red signals the localization of Lysotracker $^{\circledR}$ red DND-99. Arrows indicate pDNA not colocalized with endosomes. Scale bar $=20 \mu \mathrm{m}$.

endosomal $\mathrm{pH}$ of $5.5 .^{13)}$ In NP-N, we expect that OH-N-Chol may show single protonation at neutral $\mathrm{pH}$ due to the strong electrostatic repulsion between two protonated amines in $\mathrm{OH}-$ N-Chol.

Next, we evaluated the transfection efficiency by NP and NP-N nanoplexes into tumor cells. NP-N nanoplex exhibited lower transfection activity in PC-3, H292, Colon 26 and HeLa cells than NP nanoplex (Figs. 3A,B). These results suggested that weak association between pDNA and NP-N might cause decreased transfection activity. Therefore, we investigated whether inclusion of $\mathrm{OH}-\mathrm{N}-\mathrm{Chol}$ into NP formulation could maintain the association between pDNA and nanoparticles and increase transfection activity. NP- $0.25 \mathrm{~N}$ was prepared by substitution of $25 \% \mathrm{OH}-\mathrm{Chol}$ in NP formulation with equimolar $\mathrm{OH}-\mathrm{N}-\mathrm{Chol}$ (Table 1). NP-0.25N nanoplex was about $870 \mathrm{~nm}$ in size and had $\zeta$-potential of about $+46 \mathrm{mV}$. In gel retardation assay with NP- $0.25 \mathrm{~N}$, no migration was observed beyond a charge ratio $(+/-)$ of $3 / 1$, suggesting that $\mathrm{NP}-0.25 \mathrm{~N}$ could bind to pDNA as well as NP did (Fig. 2). In pDNA transfection, NP- $0.25 \mathrm{~N}$ increased the transfection activity with an increase of charge ratio $(+/-)$, and the transfection activity was saturated around a charge ratio (+/-) of $4 / 1$ (Fig. 3A). The transfection activity by NP- $0.25 \mathrm{~N}$ was higher than those by $\mathrm{NP}$ and NP-N in various tumor cells, and comparable to that of commercially available transfection reagent, Lipofectamine 2000 (Fig. 3B). These findings suggested that inclusion of $\mathrm{OH}-$ $\mathrm{N}$-Chol into NP formulation could increase the transfection activity by NP.

Next, we examined the cellular association of nanoplexes by flow cytometric analysis and confocal microscopy. Cellular association with NP-0.25N nanoplex of FITC-labeled pDNA at $3 \mathrm{~h}$ after transfection was significantly increased compared with that with NP or NP-N nanoplex (Fig. 4A), and the FITC- labeled pDNA transfected by NP- $0.25 \mathrm{~N}$ was strongly detected in the cells, compared with those by NP and NP-N (Fig. 4B). These results suggested that NP- $0.25 \mathrm{~N}$ could efficiently transfer pDNA into the cells by increased cellular association. The contribution of a hydroxyethyl group at the amino terminal in the enhancement of transfection has been demonstrated ${ }^{14,15}$; however, it was unclear how NP- $0.25 \mathrm{~N}$ could improve cellular association.

To determine the uptake mechanism by NP, NP- $0.25 \mathrm{~N}$ and NP-N nanoplexes in PC-3 cells, we examined the effect of endocytosis inhibitors on the cellular uptake of each nanoplex. EIPA, filipin and sucrose are inhibitors of macropinocytosis, and caveola- and clathrin-mediated endocytosis, respectively. Our results showed that sucrose treatment strongly inhibited cellular uptake by NP, NP-0.25N and NP-N (Fig. 5A), suggesting that the cellular uptake mechanism of each nanoplex was mainly clathrin-mediated endocytosis. Recently, we reported that NP-N nanoplex could efficiently deliver small interfering RNA (siRNA) into tumor cells via macropinocytosis. ${ }^{5}$ ) Although it was not clear why the uptake pathway of pDNA nanoplex was different from that of siRNA nanoplex, the difference of size between the nanoplexes of pDNA and siRNA might affect the uptake pathway.

Finally, we investigated the endosomal localization of pDNA at $24 \mathrm{~h}$ after transfection by the nanoplexes (Fig. 5B). For co-localization with endosome stained by Lysotracker, many fluorescent signals of FITC-labeled pDNA not co-localized with endosomes were observed in the cells transfected by NP- $0.25 \mathrm{~N}$ and NP-N, compared with NP. This finding indicates that NP- $0.25 \mathrm{~N}$ nanoplex might increase transfection activity by high cellular association and endosomal escape after internalization. Further study is necessary to clarify the uptake mechanism by NP- $0.25 \mathrm{~N}$. 
In this study, we prepared cationic nanoparticles, NP$0.25 \mathrm{~N}$, for pDNA delivery. The inclusion of $\mathrm{OH}-\mathrm{N}-\mathrm{Chol}$ into a nanoparticle formulation consisting of cationic cholesterol derivative could increase the transfection activity in tumor cells. However, for in vivo application, the NP-0.25N nanoplex used in this study was large in size. In future study, we will need to develop smaller nanoplex (100-200 nm) for intravenous or intratumoral injection. These findings suggested that $\mathrm{OH}-\mathrm{N}-\mathrm{Chol}$ may have potential as a helper lipid to increase the transfection for pDNA delivery.

Acknowledgements This project was supported in part by Grants-in-Aid for Scientific Research from the Ministry of Education, Culture, Sports, Science and Technology of Japan, the Advanced Research for Medical Products Mining Programme of the National Institute of Biomedical Innovation (NIBIO), and the Science Research Promotion Fund from the Promotion and Mutual Aid Corporation for Private Schools of Japan.

\section{REFERENCES}

1) Futaki S, Masui $Y$, Nakase I, Sugiura $Y$, Nakamura $T$, Kogure $K$, Harashima $\mathrm{H}$. Unique features of a $\mathrm{pH}$-sensitive fusogenic peptide that improves the transfection efficiency of cationic liposomes. $J$. Gene Med., 7, 1450-1458 (2005).

2) Slepushkin VA, Simões S, Dazin P, Newman MS, Guo LS, Pedroso de Lima MC, Düzgüneş N. Sterically stabilized pH-sensitive liposomes. Intracellular delivery of aqueous contents and prolonged circulation in vivo. J. Biol. Chem., 272, 2382-2388 (1997).

3) Hattori Y, Ding WX, Maitani Y. Highly efficient cationic hydroxyethylated cholesterol-based nanoparticle-mediated gene transfer in vivo and in vitro in prostate carcinoma PC-3 cells. J. Control. Release, 120, 122-130 (2007).

4) Yang S, May S. Release of cationic polymer-DNA complexes from the endosome: A theoretical investigation of the proton sponge hypothesis. J. Chem. Phys., 129, 185105 (2008).

5) Hattori Y, Nakamura T, Ohno H, Fujii N, Maitani Y. siRNA de- livery into tumor cells by lipid-based nanoparticles composed of hydroxyethylated cholesteryl triamine. Int. J. Pharm., 443, 221-229 (2013).

6) Igarashi S, Hattori Y, Maitani Y. Biosurfactant MEL-A enhances cellular association and gene transfection by cationic liposome. $J$. Control. Release, 112, 362-368 (2006).

7) Hattori Y, Kubo H, Higashiyama K, Maitani Y. Folate-linked nanoparticles formed with DNA complexes in sodium chloride solution enhance transfection efficiency. J. Biomed. Nanotechnol., 1, 176-184 (2005)

8) Hattori Y, Maitani Y. Folate-linked nanoparticle-mediated suicide gene therapy in human prostate cancer and nasopharyngeal cancer with herpes simplex virus thymidine kinase. Cancer Gene Ther., 12, 796-809 (2005).

9) Hattori Y, Sakaguchi M, Maitani Y. Folate-linked lipid-based nanoparticles deliver a NFkappaB decoy into activated murine macrophage-like RAW264.7 cells. Biol. Pharm. Bull., 29, 1516-1520 (2006).

10) Sakurai F, Inoue R, Nishino Y, Okuda A, Matsumoto O, Taga T, Yamashita F, Takakura Y, Hashida M. Effect of DNA/liposome mixing ratio on the physicochemical characteristics, cellular uptake and intracellular trafficking of plasmid DNA/cationic liposome complexes and subsequent gene expression. J. Control. Release, 66 , 255-269 (2000).

11) Ross PC, Hui SW. Lipoplex size is a major determinant of in vitro lipofection efficiency. Gene Ther., 6, 651-659 (1999).

12) Turek J, Dubertret C, Jaslin G, Antonakis K, Scherman D, Pitard B. Formulations which increase the size of lipoplexes prevent serumassociated inhibition of transfection. J. Gene Med., 2, 32-40 (2000).

13) Han M, Bae Y, Nishiyama N, Miyata K, Oba M, Kataoka K. Transfection study using multicellular tumor spheroids for screening non-viral polymeric gene vectors with low cytotoxicity and high transfection efficiencies. J. Control. Release, 121, 38-48 (2007).

14) Percot A, Briane D, Coudert R, Reynier P, Bouchemal N, Lièvre N, Hantz E, Salzmann JL, Cao A. A hydroxyethylated cholesterolbased cationic lipid for DNA delivery: effect of conditioning. Int. J. Pharm., 278, 143-163 (2004).

15) Hasegawa S, Hirashima $N$, Nakanishi M. Comparative study of transfection efficiency of cationic cholesterols mediated by liposomes-based gene delivery. Bioorg. Med. Chem. Lett., 12, 1299-1302 (2002). 